\title{
A Multi-objective Optimal Dispatch Method for Integrated Energy System Considering Multiple Loads Variations
}

\author{
Dong Lv ${ }^{1, a}$, Jun Tang ${ }^{1, b}$ and Chen Yang ${ }^{1, c}$ \\ ${ }^{1}$ State Grid Suzhou Power Supply Company, Suzhou, Jiangsu Province, China
}

\begin{abstract}
This paper proposes a multi-objective optimization dispatching method for the Integrated Energy System (IES), in which cooling/heating/electricity multi-load demand are included, and IES's requirements in terms of energy efficiency, economy, and emissions are considered. We established an IES model for multi-objective optimal dispatch, and simulated cooling/heating/electric multiple load variations in it; then we established multiple objective functions based on IES's scheduling requirements in terms of energy efficiency, economy, environmental issues. The model is solved by finding the pareto front based on genetic algorithm (GA), so that the decision makers can filter out the scheduling plan based on their preference, in which single-objective optimization is used first to find out the initial population for GA. Finally, the proposed method's effectiveness is verified by case studies.
\end{abstract}

\section{INTRODUCTION}

With global energy crisis and environmental problems, the world has begun to promote the energy transition and build the integrated energy system (IES) with multi-energy systems optimized as a whole. Energy Internet, with large-scale utilization of distributed renewable energy at the physical layer, could realize the synergy and integration of multiple energy systems such as power grid, transportation system, natural gas pipelines, heating/cooling network, etc. 1, which is useful for improving energy utilization. It is of great significance to achieve the overall efficiency of clean and renewable energy, realize the large-scale utilization of clean and renewable energy, and fully reveal the flexibility of the energy system to address the uncertainties issue of renewable energy. IES involves the multi-energy coupling of cooling, heating, electricity, and natural gas, and includes a variety of energy-supply facilities, which makes the overall system operation and configuration much more complicated than conventional energy system. Moreover, the development of modern smart grids and Energy Internet technology has an impact on the economy and energy saving of microgrids and IES, where environmental protection and other indices have been included to realize higher requirements 2 . It can be seen that the optimal dispatch for IES operation needs to be comprehensively optimized from different perspectives such as energy efficiency, economy, and environment. How to establish a suitable multi-objective optimization scheduling model, as well as the solution algorithm, has become some of the major concerns.

Currently, the multi-objective optimal dispatch methods could be divided into two categories: 1)
Converted it into a single-objective optimization problem by giving each objective function a weight coefficient, the single-objective problem can be then optimized by state-of-the-art algorithms and software; 2) Solve the multi-objective optimization problem directly, namely obtain the Pareto Front (PF), and use it as a reference to select the optimal dispatch plan. For the first category, some scholars have adopted the interior point method, fuzzy optimization theory [3], analytic hierarchy process [4], etc. to carry out the research of combining multiple objectives into a single objective, and try to combine economics [5] and environment [3], security [6] [7] and other indicators are modeled as corresponding objective functions to guide the system's multi-objective optimal dispatch. For the second category, there has been less progress in the related research, mainly due to the more complex IES structure. Many scholars have realized the accurate calculation of PF by studying more efficient heuristic algorithms. However, as convex relaxation and other technologies are applied in power systems [11][12], the establishment of a convex optimization model through the convex relaxation of the IES model or other methods of convexity of the model to simplify the PF solution is also a key direction. In terms of the improvement of the optimization algorithm, Musau et al. [13] used simulated annealing algorithm to solve the economic dispatch model of the thermoelectric coupling system including thermal energy and renewable energy on multi-objective such as line loss and emission. K.C. Kavvadias et al. [14] applied the multi-objective optimization algorithm to the combined cooling, heating and power system (CCHP), and finally obtained the optimal device capacity, operation strategy and optimal device capacity.

In this context, this paper proposes an IES multi-objective optimal dispatch model and corresponding

aemail: carruler@163.com, ${ }^{\mathrm{b} e m a i l: ~ p r e c k e t 2003-1 v @ 163 . c o m, ~}{ }^{\mathrm{c} e m a i 1:}$ 232267346@qq.com 
solution method, which considers energy efficiency, economy, environmental issues in the objective functions. IES optimal dispatch is formulated as a linear programming problem and solved by genetic algorithm, in which some initial populations are firstly obtained by solving the IES model with single one objective function, thus improving the PF's quality. Finally, this paper applies the corresponding method to a real-world IES to verify the effectiveness of proposed method.

\section{MODEL FORMULATION FOR IES}

This paper adopts methods such as convex model to ensure the convexity of the IES mathematical model. At the same time, this paper fully considers the IES composition structure, operating conditions, and coupling relationships between different equipment, such as equipment operating constraints, climbing ability, and multi-energy coupling relationships, etc. The linear model of the combined cooling heating and power (CCHP) system used in this paper is as follows:

$$
\begin{gathered}
P_{c, \min } \leq P_{c}(i) \leq P_{c, \max } \\
P_{c, d n} \leq P_{c}(i+1)-P_{c}(i) \leq P_{c, u p} \\
H_{c, \min } \leq H_{c}(i) \leq H_{c, \max } \\
H_{c, d n} \leq H_{c}(i+1)-H_{c}(i) \leq H_{c, u p} \\
a_{1} P_{c}(i)+b_{1} H_{c}(i)=F_{c}(i) \\
H_{c}(i)=c_{1} P_{c}(i)+d_{1} \\
C_{c, \min } \leq C_{c}(i) \leq C_{c, \max } \\
C_{c, d n} \leq C_{c}(i+1)-C_{c}(i) \leq C_{c, u p} \\
a_{2} P_{c}(i)+b_{2} C_{c}(i)=F_{c}(i) \\
C_{c}(i)=c_{2} P_{c}(i)+d_{2}
\end{gathered}
$$

Where: $i$ is the time, $i \in T$; $T$ is the total time; $P_{c, \min }$ and $P_{c, \max }$ are the minimum and maximum values of CCHP electric power; $H_{c, \min }$ and $H_{c, \max }$ are the minimum and maximum values of CCHP heating power; $C_{c, \min }$ and $C_{c, \text { max }}$ are the minimum and maximum values of CCHP cooling power. $P_{c}(i), H_{c}(i)$ and $C_{c}(i)$ are the electric power, heating power, and cooling power of CCHP at time $i$; $P_{c, u p}$ and $P_{c, d n}$ are the upper and lower bounds of the CCHP electric power climbing ability; $H_{c, u p}$ and $H_{c, d n}$ are the upper and lower bounds of the CCHP heating power climbing ability; $C_{c, u p}$ and $C_{c, d n}$ are the upper and lower bounds of CCHP cooling power climbing ability; $F_{c}(i)$ is the power of gas consumed by CCHP at time $i ; a_{l}, b_{l}$, $c_{1}, d_{1}, a_{2}, b_{2}, c_{2}$ and $d_{2}$ are the corresponding efficiency coefficients.

Constraints (1) (3) (7) respectively limit the upper and lower bounds of CCHP cooling and heating electric power, (2) (4) (8) are the climbing constraints of CCHP, (5) (6) (9) (10) explain the energy conversion situation in the unit.

The remaining equipment in IES, such as gas turbines, heat pumps, electric heating equipment, electric refrigeration equipment, adopt convex models to ensure the global optimal solutions.

\section{MODEL FORMULATION FOR OBJECTIVE FUNCTIONS}

\subsection{Energy efficiency objective function}

IES can realize the efficient coupling of multi-energy complementary integrated energy systems. Therefore, how to establish a reasonable objective function to realize the energy-efficient dispatch operation of IES is extremely important when optimizing dispatch.

When evaluating energy efficiency, the general formula for calculating energy efficiency based on the first law of thermodynamics is: energy efficiency is equal to the sum of the total amount of various energy consumed by the terminal divided by the total amount of primary energy consumed. The expression of energy efficiency is as shown as (11). The analysis shows that the energy utilization efficiency can evaluate the utilization rate of energy in quantity, and the higher energy utilization rate indicates that the system can maximize the utilization of multiple energy sources during operation.

$$
\eta=\frac{\sum_{t=1}^{T}\left[L_{\mathrm{e}}(t)+L_{h}(t)+L_{c}(t)\right]}{\sum_{t=1}^{T}\left[P_{\text {wind }}(\mathrm{t})+P_{p v}(t)+P_{h y d r o}(t)+P_{C C H P}(t)\right]}
$$

Where: $P_{\text {wind }}(t)$ is the power generated by wind; $P_{p v}(t)$ is the power generated by photovoltaics; $P_{\text {hydro }}(t)$ is the power generated by hydropower; $P_{C C H P}(t)$ is the power generated by CCHP; $L_{c}(t)$ is the cooling load of the system, which can be calculated by the flow, temperature, pressure, and heating value of the cooling gas; $L_{h}(t)$ is the heating load of the system, which can be calculated from the flow, temperature, pressure and calorific value of the heating steam; $L_{e}(t)$ is the electrical load of the system; $T$ is the total time.

\subsection{Economic objective function}

In the IES dispatch operation problem, the equipment operating cost is the main component of the economic objective function, which mainly includes the IES's external power grid purchase cost, gas purchase cost, subsidy situation $M_{f}$, along with the system operation and maintenance $\operatorname{cost} M_{o}$ :

$$
\begin{gathered}
M_{\mathrm{f}}=\sum_{k}^{K} \sum_{i}^{T} M_{\text {gas }, \text { elec }, k}(i) C_{\text {gas }, \text { elec }, k} P_{\text {gas }, \text { elec }, k} \\
M_{\mathrm{o}}=\sum_{k}^{K} \sum_{i}^{T} M_{\text {run }, k}(i) P_{k}
\end{gathered}
$$

Where: $k$ is the number of each component of the system, $K$ is the total number of equipment in the system; $M_{\text {gas, elec, } k}(i)$ is the price of natural gas purchase, power purchase from the external grid, and new energy/CCHP power generation subsidies for the unit capacity of component $k$ at time $i$; Correspondingly, $C_{\text {gas,elec, } k}$ is the natural gas consumption of each component $k$ of the system in unit time $i$, electricity purchased from external networks, and new energy/CCHP power generation; 
$P_{\text {gas,elec, } k}$ is the capacity of component $k ; M_{\text {run, }}(i)$ is the operation and maintenance cost of unit capacity of each component $k$ of the system in unit time; $P_{k}$ is the rated capacity of component $k$.

\subsection{Ecological objective function}

IES pollutant emissions and carbon emissions are key to environmental assessment. This paper first calculates the corresponding pollutant emissions of the system, mainly considering the atmospheric acidification effect of N20, $\mathrm{SO} 2, \mathrm{NOx}$ and other gases, and the greenhouse caused by $\mathrm{CH} 4$ and $\mathrm{CO} 2$. Then the dust effect caused by PM2.5 is estimated. Next, this paper quantitatively assesses the environmental impact of the pollutants, such as atmospheric acidification, climate warming, and increase in dust particles. The calculation method of the emission amount of the $m$-th pollutant and the quantified IES environmental assessment result $W$ is as follows:

$$
\begin{gathered}
C_{m}=\sum_{i}^{T} \mu_{m} C_{g a s}(i) \\
W=w_{1} \sum_{m}^{M} \phi_{m} C_{m}+w_{2} \sum_{m}^{M} \rho_{m} C_{m}+w_{3} \sum_{m}^{M} \theta_{m} C_{m}
\end{gathered}
$$

Where: $\mu_{m}$ is the emission coefficient of different types of pollutant gases when natural gas is used; $m$ is the pollutant number, $m \in M, M$ is the total number of equipment in the system; $C_{g a s}(i)$ is the natural gas consumption per unit time $i ; w_{1}, w_{2}$ and $w_{3}$ are the weighting factors for the three effects of atmospheric acidification, climate warming, and dust particle increase caused by pollutants, which can be determined according to actual evaluation requirements; $\phi_{m}$ is the effect factor for generating atmospheric acidification for the $m$-th pollutant; $\rho_{m}$ is the $m$ th The effect factor of class pollutants to produce climate warming; $\theta_{m}$ is the effect factor of pollutants of class $\mathrm{m}$ to increase dust particles.

This article assumes that the potential value of $\mathrm{CO}_{2}$ to cause climate warming is defined as 1 , then the $\mathrm{CO}_{2}$ equivalent value of different greenhouse gases emitted in a certain period can be calculated. In the same way, the potential value of $\mathrm{SO}_{2}$ to cause atmospheric acidification is defined as 1 . The value of the potential for causing dust particles to increase by $P M_{2.5}$ is defined as 1 . The detailed parameters are shown in Table 1. According to the potential value of different gases to cause atmospheric acidification, climate warming, and increase of dust particles, quantified values of three effect factors can be obtained.

Table 1 The coefficients of pollution

\begin{tabular}{cccc}
\hline Pollutant & $\begin{array}{c}\rho_{m} \text { (g } \\
\mathrm{CO}_{z} \text {-equiv.) }\end{array}$ & $\begin{array}{c}\phi_{m} \text { (g } \\
\mathrm{SO}_{z} \text {-equiv.) }\end{array}$ & $\begin{array}{c}\phi_{m} \text { (g } \\
\mathrm{PM}_{2.5} \text {-equiv) }\end{array}$ \\
\hline $\mathrm{CO}_{2}$ & 1 & - & - \\
$\mathrm{CH}_{4}$ & 21 & - & - \\
$\mathrm{N}_{2} \mathrm{O}$ & 310 & 0.7 & -
\end{tabular}

\begin{tabular}{cccc}
$\mathrm{SO}_{2}$ & - & 1 & 1.9 \\
$\mathrm{NO}_{x}$ & - & 0.7 & 0.3 \\
$\mathrm{PM}_{2.5}$ & - & - & 1 \\
\hline
\end{tabular}

\section{MULTI-OBJECTIVE OPTIMAL DISPATCH MODEL OF IES}

\subsection{0bjective function}

The energy-saving, economical, and environmentally friendly features of IES are three contradictory objective functions. Since the changes of the three are related to the operating conditions of multiple units in the system, there is not a simple correlation between every two objective functions. Through multi-objective optimization problem description. Integrating the energy efficiency, economy, and ecological objective functions established above, the objective function of the IES multi-objective optimization scheduling problem can be obtained:

$$
\begin{gathered}
\min \mathbf{f}=\left(F_{1}, F_{2}, F_{3}\right) \\
F_{1}=1 / \eta \\
F_{2}=M_{\mathrm{f}}+M_{\mathrm{o}} \\
F_{3}=W
\end{gathered}
$$

Where: $F_{1}$ is the economic objective function; $F_{2}$ is the environmental objective function; $F_{3}$ is the energy efficiency objective function.

It can be seen that when the IES cooling, heating, electric load power and the temperature of the cooling and heating working medium are known, it is a non-convex function. The reciprocal is used as the objective function of energy efficiency to ensure the convexity of the model. Because the improvement of IES economy, energy saving, and environmental protection are all related to the smaller value of the objective function $F_{1}, F_{2}, F_{3}$, a unified vector optimization problem for finding the minimum can be established.

\subsection{Constraints}

The established constraints of the multi-objective optimization problem mainly include equipment output constraints, equipment climbing constraints and load balance constraints. The equipment output constraints and equipment climbing constraints are explained in detail in the preceding mathematical model, mainly to constrain the upper and lower bounds of the output of each unit in the optimal scheduling problem and the upper and lower bounds of the unit output changes in adjacent time periods. Load balance constraints mainly include electric load, heating load and cooling load balance. Load balance allows a certain margin, and the equation is relaxed into the following form:

$$
\begin{gathered}
P_{\mathrm{c}}(\mathrm{t})+P_{\text {wind }}(t)+P_{\mathrm{pv}}(t)+P_{\text {hydro }}(t) \geq L_{\mathrm{e}}(t) \\
H_{\mathrm{c}}(t)+P_{\text {boil }}(t)+P_{\text {eth }}(t) \geq L_{\mathrm{h}}(\mathrm{t}) \\
C_{\mathrm{c}}(\mathrm{t})+P_{\text {etc }}(t) \geq L_{\mathrm{c}}(t)
\end{gathered}
$$




\section{Solving Method of Multi-Objective Optimal Dispatch PRoblem}

The optimal scheduling of IES is a constrained optimization problem. For example, directly calling traditional heuristic algorithms, such as genetic algorithms, cannot guarantee that a good quality initial feasible solution can be obtained, and then a Pareto frontier can be obtained. In this regard, this paper designs a two-stage solution method. First, through the optimization of different objective functions, a set of initial feasible solutions is found as the initial population of the genetic algorithm; then, the genetic algorithm is called to solve the problem, and the quality is finally obtained. High Pareto frontiers, power system decision makers make choices. The method flow chart is as follows:

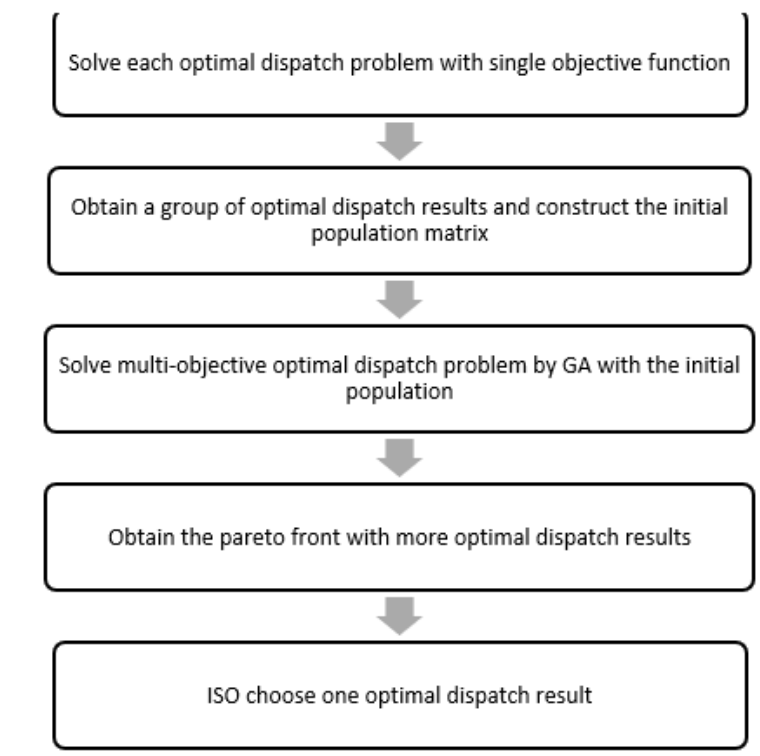

Fig. 1 The Proposed Framework for Multi-objective IES Optimal Dispatch

\subsection{The first stage: Solve the single objective optimization problem}

Since multi-objective optimization problem (MOOP) is also considered to belong to the vector optimization problem, scalarization of the vector-like objective function has been one of the methods to solve a MOOP and find the PF. Using scalarization, we can find Pareto optimal points for any vector optimization problem by solving the ordinary scalar optimization problem. In this regard, what we've been doing in the first stage, is trying to find the optimal points with different $\lambda \succeq_{K^{*}} 0$, where $\lambda^{\mathrm{T}} \boldsymbol{f}(x)$ is the scalarized objective function of MOOP, stated as below:

$$
\begin{gathered}
\min \lambda^{\mathrm{T}} \boldsymbol{f}=\lambda_{1} F_{1}(x)+\lambda_{2} F_{2}(x)+\lambda_{3} F_{3}(x)+\cdots \\
\text { s.t. } \quad f_{i}(x) \leq 0, i=1, \cdots, m \\
h_{i}(x)=0, i=1, \cdots, p
\end{gathered}
$$

For instance, assuming we have 3 objectives in IES optimal dispatch problem, by using
$\lambda_{1}=(1,0,0)^{\mathrm{T}} \quad, \quad \lambda_{2}=(0,1,0)^{\mathrm{T}}$ and $\lambda_{3}=(0,0,1)^{\mathrm{T}}, \quad$ we solve single-objective optimization problem with $\lambda_{1}^{\mathrm{T}} \boldsymbol{f}(x)$, $\lambda_{1}^{\mathrm{T}} \boldsymbol{f}(x)$ and $\lambda_{1}^{\mathrm{T}} \boldsymbol{f}(x)$ as objective function, respectively, and obtain the optimizer $x_{1}^{*}, x_{2}^{*}$ and $x_{3}^{*}$, then these solutions can be used to construct the initial population matrix for GA.

\subsection{The second stage: Solve multi-objective optimization problems through genetic algorithm}

With the high-quality initial population (solutions), we can then solve the multi-objective optimal dispatch problem by Genetic Algorithm (GA). GA is a search-based optimization technique based on the principles of genetics and natural selection. In GA, with a population of candidate solutions to an optimization problem is evolved toward better solutions. Once the genetic representation and the fitness function are defined, a GA proceeds to initialize a population of solutions and then to improve it through repetitive application of the mutation, crossover, inversion and selection operators. However, GA often faces difficulties in constrained optimization problem because the mutation and crossover could generate infeasible solutions if there are many constraints in the problem. That's exactly what a day-ahead optimal dispatch problem has, especially for IES integrated with multiple load variations and energy-supply facilities.

\section{CAse Study}

This paper takes a IES project in China as an example, to analyze the effectiveness of objective function modeling, the overall model and the significance of multi-objective optimal dispatch.

The regional IES is mainly composed of wind power, photovoltaic power, small hydropower, CCHP, heat storage devices, heat pumps, high-speed rail stations, hospitals, external power grids, charging stations, etc. CCHP provides cooling/heating by connecting a lithium bromide unit. According to the working principle of the lithium bromide unit, the heating efficiency of CCHP is the same as the cooling efficiency, and both are thermal efficiency. The operating parameters of the lithium bromide absorption refrigeration unit are as follows: the exhaust temperature of the unit is $120^{\circ} \mathrm{C}$, the make-up water temperature is $18^{\circ} \mathrm{C}$, the hot water temperature when the unit is heating $65^{\circ} \mathrm{C}$, and the cold water temperature when the unit is cooling $7^{\circ} \mathrm{C}$. In this paper, the illumination time is set to 7:00-18:00, the low calorific value of natural gas is $37.85 \mathrm{MJ} \cdot \mathrm{Nm} 3$, the conversion value of natural gas is $0.0989 \mathrm{Nm} 3 / \mathrm{kWh}$, the peak electricity price is 1.438 yuan/kWh, the valley price is 0.3 yuan $/ \mathrm{kWh}$, and the average electricity price $0.5 \mathrm{yuan} / \mathrm{kWh}$, gas price is 3.79 yuan/ $\mathrm{Nm} 3$; the subsidies considered are CCHP subsidy 0.025 yuan $/ \mathrm{kWh}$, wind power subsidy 0.2 yuan/kWh, photovoltaic subsidy 0.32 yuan $/ \mathrm{kWh}$; natural 
gas and chemical fuel cell emission coefficients are 204.255 respectively $\mathrm{kg} / \mathrm{MWh}, 1.554 \mathrm{~kg} / \mathrm{MWh}$.

\subsection{The Pareto Front End of the System and the Solving of the Multi-Objective Optimal Scheme}

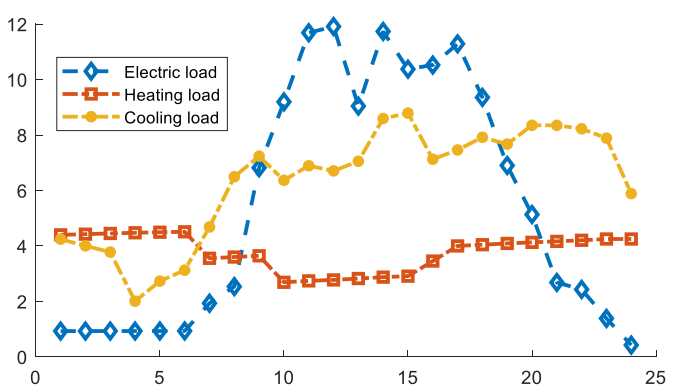

Fig.2 The varying multiple loads of a typical day

In view of the situation of IES in Guizhou, an optimal dispatch plan for IES in a typical day is selected. Typical daily load fluctuations are shown in Figure 2. PF is shown in Figure 3.

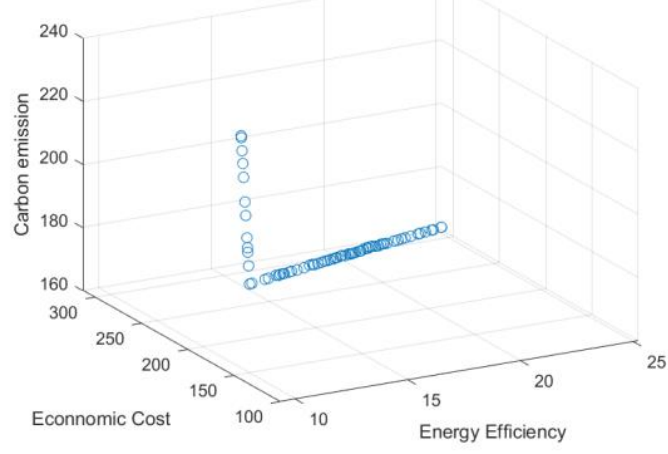

Fig.3 The Pareto Front of IES Optimal Dispatch

\subsection{Analysis of Multi-Objective Optimal Dispatch Results Selected by Pareto Front End}

This paper selects three typical multi-objective optimization scheduling results from the Pareto front end, compares the characteristics of the system optimization scheduling results led by different objective functions, compares the differences of the obtained scheduling results. Then this paper explains the effectiveness of function modeling and the significance of system energy efficiency as an energy efficiency objective function.

The values of the different objective functions of the obtained scheduling results are:

Table 2 The Objectives of Different Cases

\begin{tabular}{|l|c|c|c|}
\hline \multirow{2}{*}{ Objectives } & \multicolumn{3}{|c|}{ Case Number } \\
\cline { 2 - 4 } & Case 1 & Case 2 & Case 3 \\
\hline $\begin{array}{l}\text { Objective 1 } \\
\text { (Efficiency) }\end{array}$ & 9.30 & 141.5 & 237.3 \\
\hline $\begin{array}{l}\text { Objective 2 } \\
\text { (Economy) }\end{array}$ & 9.48 & 136.9 & 190.9 \\
\hline $\begin{array}{l}\text { Objective 3 } \\
\text { (Emission) }\end{array}$ & 25.22 & 312.5 & 160.7 \\
\hline
\end{tabular}
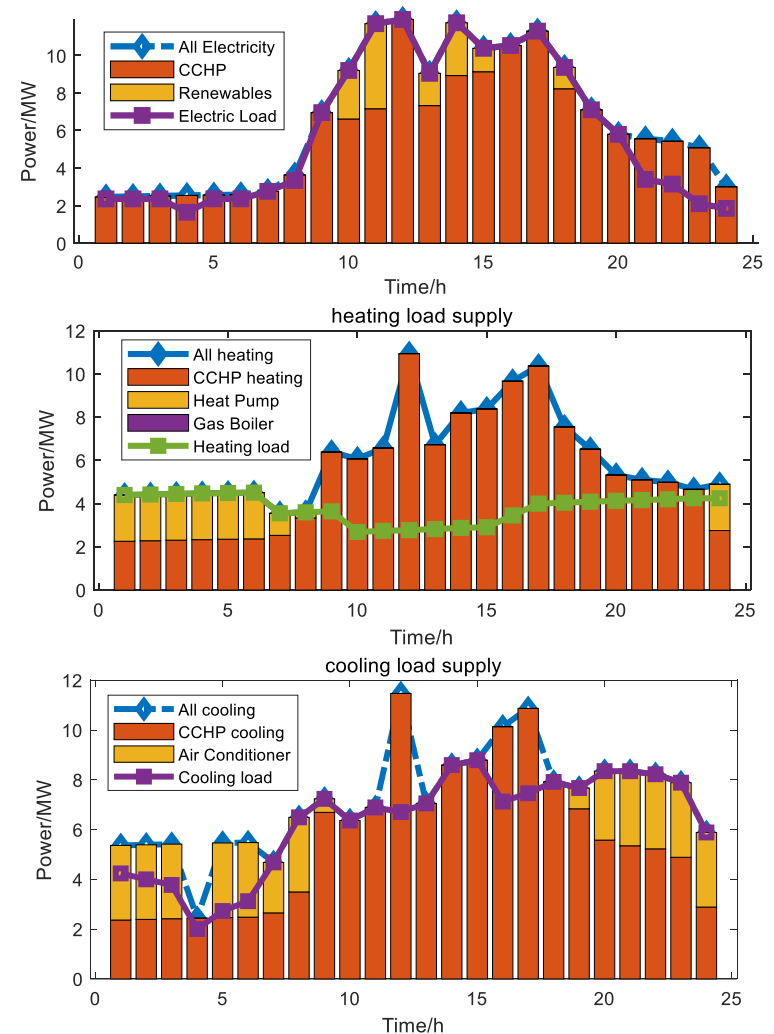

(a)
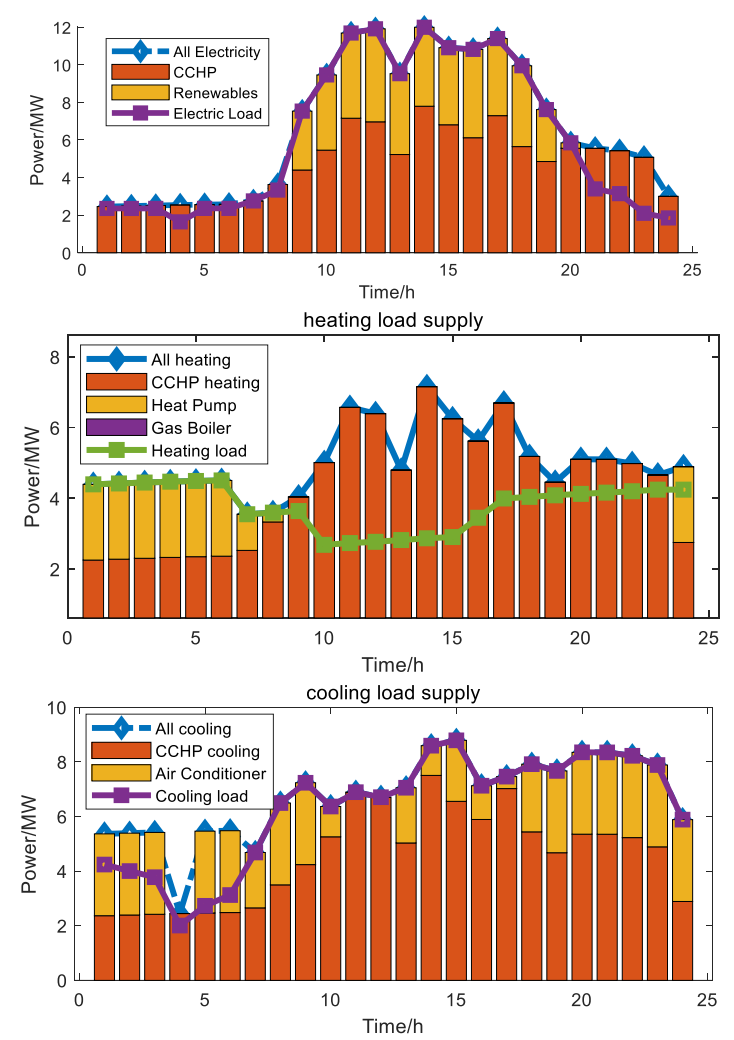

(b) 

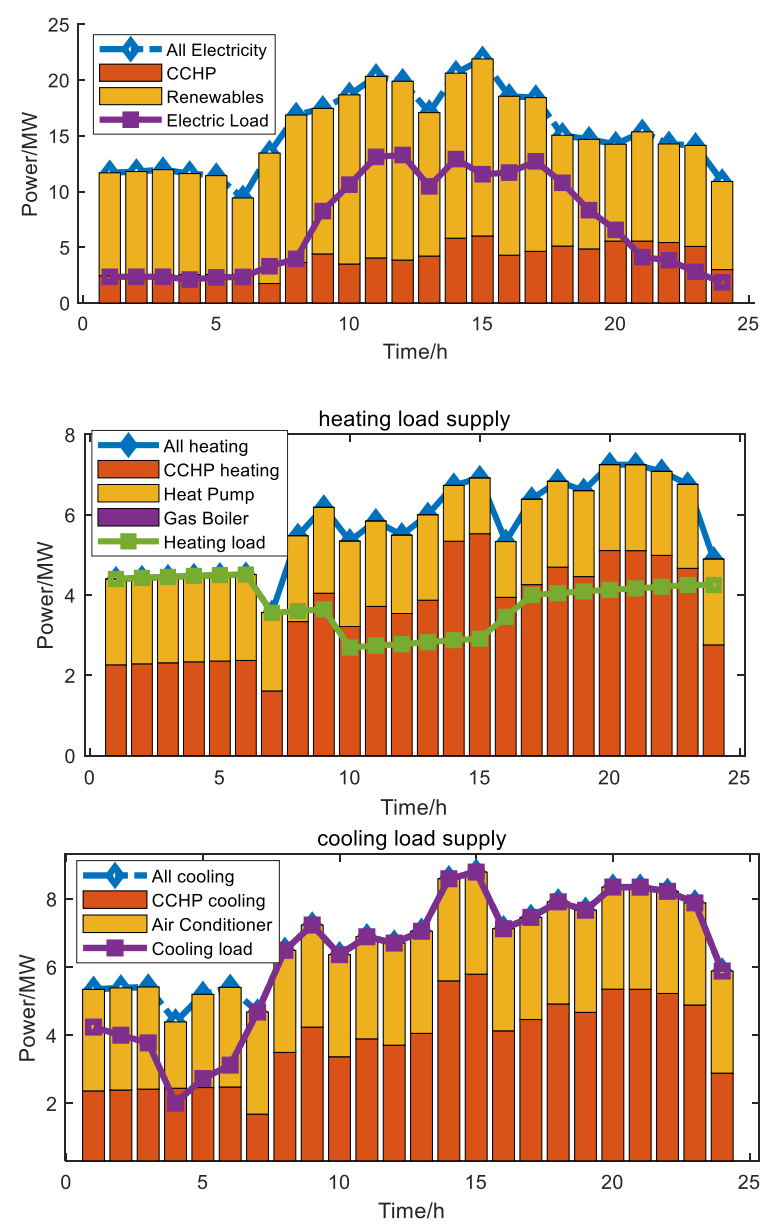

(c)

Fig.4: The optimal dispatch plan in (a)case 1; (b) case 2; (c) case 3

Comparing the results of Figure 3, it can be seen that in the optimization with the energy efficiency objective function as the main objective function in case 1 , the energy efficiency objective function (i.e. $F_{l}$ ) can reach the optimal minimum; Similarly, in case 2 and 3 , the economic objective function and the environmental objective function can respectively reach the optimal minimum.

Making further analysis according to data in Figure 3, the following conclusions can be obtained:

(1) Comparing the economic objective function as the main objective function (case 2) and environmental objective function as the main objective function (case 3 ), the output of CCHP in the latter has decreased, while the output of wind power, photovoltaic energy and hydropower has increased. This is mainly because CCHP is relatively economical, and the natural gas it consumes has certain pollutant emissions, which is bad for environmental protection. At the same time, new energy has zero emissions, and is good for environmental protection.

(2) Comparing case 1 and 3, when the energy efficiency objective function is dominant in the multi-objective problem, the output of CCHP increases, which is mainly because of its higher energy utilization and conversion rate. Due to the increase in output of CCHP, it can simultaneously meet the needs of the cooling, heating, and electrical loads in the system. Meanwhile, the output of other units such as corresponding hydropower new energy sources has decreased.

The analysis of comprehensive cases shows that because CCHP has corresponding policy subsidies, it has good economic efficiency, can realize the hierarchical utilization and high-quality utilization of energy, and can improve the energy efficiency of the system. However, it has poor environmental protection due to the emission of pollutants. Different from CCHP, new energy sources has fewer subsidies and pollutants, so it is more environmentally friendly.

When the system performs multi-objective dispatch, the dispatch plan of each unit will be different considering different requirements. The PF in Figure 2 can provide a dispatch plan under any combination of objective function weights. According to the different requirements for energy efficiency, economy, and environment, further constraints can be added to limit the range of the objective function, and the PF can offer solutions to meet the need. According to the optimal dispatch results, it can be found that the multi-objective optimal dispatch plan can balance the optimal dispatch options among multiple objectives. The final dispatch plan can make the system operate in an more reasonable condition with different requirements satisfied.

\section{ConClusions}

Facing the challenges of global climate and energy changes, it is very important to make full use of IES to effectively integrate the original energy supply systems, and to achieve high-efficiency, energy-saving and economic operation of multi-energy coupling system. This paper proposes a multi-objective optimal dispatch model for IES and its corresponding solution method, which comprehensively considers the optimal dispatch requirements in terms of energy efficiency, economy, and environment, and an initial population is firstly obtained by solving single-objective. According to the comparison of existing research and cases, it is found out that IES can obtain a group of optimal dispatch plan to meet the requirements of the system in terms of energy efficiency, economy, and environment. Moreover, the quality of solutions is significantly improved by solving single-objective optimal dispatch firstly and constituting an initial population for GA.

\section{References}

1. SUN Hongbin, et al. Integrated Energy Management System: Concept, Design, and Demonstration in China [J]. IEEE Electrification Magazine, 2018, 6(2): 42-50.

2. JIA Hongjie, et al. Thought About the Integrated Energy System in China[J]. Electric Power Construction, 2015, 36(1):16-25. 
3. CHEN, J., et al. Microgrid multi-objective economic dispatch optimization $[\mathrm{J}]$. Proceedings of the CSEE, 33(19): 57-66.

4. JIANG Youyin, et al. A fuzzy multi-criteria decision making model for CCHP systems driven by different energy sources[J]. Energy policy, 2012(42): 286-296. doi: 10.1016/j.enpol.2011.11.085

5. WONG KP, et al. Multi-objective generation dispatch using bi-criterion global optimization[J]. IEEE Transactions on Power Systems, 1993, 10(4): 1813-1819.

6. LIN, Shunjiang, et al. Normalised normal constraint algorithm applied to multi-objective security-constrained optimal generation dispatch of large-scale power systems with wind farms and pumped-storage hydroelectric stations[J]. IET Generation, Transmission \& Distribution, 2017, 11(6): 1539-1548.

7. HAMIDI Amir, et al, D-FACTS Cooperation in Renewable Integrated Microgrids: A Linear Multi-Objective Approach[J], IEEE Transactions on Sustainable Energy, 2017.

8. GAN Lingwen, et al. Exact Convex Relaxation of Optimal Power Flow in Radial Networks[J]. IEEE Transactions on Automatic Control, 2015, 60(1): 72-87.
9. LAVAEI Javad, et al. Zero Duality Gap in Optimal Power Flow Problem[J]. IEEE Transactions on Power Systems, 2012, 27(1): 92-107.

10. MUSAU, M. p., et al. Multi area multi objective dynamic economic dispatch with renewable energy and emissions [C]. IEEE International Energy Conference (ENERGYCON), April, 4-8, 2016, Leuven, Belgium.

11. KAVVADIAS K. C., et al. Multi-objective optimization of a trigeneration plant $[\mathrm{J}]$. Energy Police, 2010(38): 945-954.

12. LI Zhengshuo, et al. Storage-like devices in load leveling: Complementarity constraints and a new and exact relaxation method[J]. Applied Energy, vol.151, no. 1 , pp. 13-22.

13. BIE Xiang and HAN Guangze. Thermodynamic analysis of natural gas fired combined cooling heating and power system[J], Chemical Engineering (China), 2010. 38(1): 57-62.

14. CHEN Cong, et al. A multi-objective evaluation method for distributed integrated energy system[C]. IEEE Conference on Energy Internet and Energy System Integration (EI2), Nov 26-28, 2017, Beijing, China. 